\title{
Analysis of the Treatment Efficacy of Percutaneous Catheter Drainage Under CT Guidance for Severe Acute Necrotizing Pancreatitis
}

\author{
Xin $\mathrm{Xu}^{1,2}$, Xuening Zhang ${ }^{1,{ }^{*}}$ and Honggen Liu ${ }^{3}$ \\ ${ }^{1}$ Department of Radiology, The Second Hospital of Tianjin Medical University, Tianjin, China \\ ${ }^{2}$ Department of Radiology, Tianjin Hospital of ITCWM, Nankai Hospital, Tianjin, China \\ ${ }^{3}$ Department of Oncology, Tianjin Medical University Cancer Hospital, Tianjin, China \\ "Corresponding author: Department of Radiology, The Second Hospital of Tianjin Medical University, Tianjin, China. Email: lucyxn2016@126.com
}

Received 2018 April 01; Revised 2018 November 20; Accepted 2018 November 27.

\begin{abstract}
Background: Percutaneous catheter drainage (PCD) therapy is an important treatment for severe acute pancreatitis.

Objectives: The purpose of this retrospective study was to analyze the relevant risk factors of computed tomography (CT) guided PCD during the treatment of infected pancreatic necrosis (IPN) in severe acute pancreatitis.

Patients and Methods: This is a retrospective study. From January 2013 to November 2016, 162 patients suffering from severe acute pancreatitis with IPN were assessed using CT-guided PCD. Abdominal CT scan was performed for the patients. The interventional therapist chose the location and puncture according to the image. Depending on the efficacy and process of the treatment, these patients were divided into a PCD success treatment group and a PCD combined with surgery group. Factors affecting the success of PCD treatment were analyzed by logistic regression analysis.

Results: Among the 162 cases, 71 cases (43.82\%) were in the PCD success group and 91 cases (56.17\%) were in the PCD combined with surgery group. Through the course of treatment, CT values of piercing zone, acute physiology and chronic health evaluation II (APACHEII) scores and modified CT severity index (MCTSI) scores showed statistically significant correlation with the therapeutic effect of PCD under CT guidance. A further multivariate analysis found that the CT value of puncture area is the best predictor for efficacy and when the CT value got higher the PCD efficacy would become lower. We performed further analysis of the factors associated with the average CT value in the puncture area which was higher than 20 Hounsfield unit (HU), and found that the length of time from patient admission to drainage, APACHEII scores, MCTSI scores and C reactive protein (CRP) levels were risk factors for PCD treatment efficacy.

Conclusion: CT values of piercing zone is the major risk factor affecting the curative effect of CT guidance PCD. For patients with higher CT values in the puncture area, the longer time from patient admission to drainage, the higher APACHEII scores and MCTSI scores. Higher levels of $\mathrm{C}$ reactive protein seem to lower the curative effect.
\end{abstract}

Keywords: Severe Acute Pancreatitis, CT Guided, Percutaneous Catheter Drainage

\section{Background}

Acute pancreatitis (AP) is a common clinical disease demonstrating acute abdominal symptoms. During the course of the disease, pancreatic enzymes are activated and the pancreatic tissue is corroded. In 2013, the international pancreatitis research institutions revised the Atlanta classification. Pancreatitis can be divided into mild pancreatitis, moderately severe pancreatitis and severe pancreatitis. The mild acute pancreatitis is self-limiting. Moderately severe acute pancreatitis can cause brief organ failure, which is easy to correct and the mortality is lower. Severe acute pancreatitis is more dangerous and can be accompanied by many complications such as intraabdominal hypertension (IAH). IAH can cause a series of pathophysiological changes in the body and damage the respiratory system, circulatory system and urinary system, even leading to abdominal compartment syndrome (ACS) (1).

Although the diagnostic and treatment technology for AP has developed rapidly, the prognosis of acute severe pancreatitis is still poor. A debridement surgery performed too early for necrotizing pancreatitis can aggravate the symptoms. The trauma from laparotomy is bigger, in which the incidence of postoperative complications is approximately $34 \%-95 \%$ and the mortality is up to $11 \%-39 \%$ (2-5).

Infected pancreatic necrosis (IPN) accounts for 33\% of necrotizing pancreatitis (6). The mortality in patients with 
IPN is about 32\% (7). In recent years, a consensus has been basically reached as to the timing of IPN treatment. It is generally believed that the surgical intervention should be performed about 4 weeks after the onset of the symptoms $(8,9)$. The "step-up" method is an emerging treatment concept. Percutaneous catheter drainage (PCD) usually takes a small incision on the surface of the body, and then goes retrograde into the lesion along the insertion path, and then the necrotic tissue is removed. Because of the small trauma and easy operation, it is usually used as the initial treatment for IPN (10-13). Many studies have reported the superiority of PCD to necrosectomy (14-20). Some scholars reported that during the treatment of patients with IPN, PCD has cured a considerable number of patients without the need for removal of necrotic tissue by operation (13). Sometimes PCD is used as a transitional measure, while an effective antibiotic therapy can delay the surgical time for patients requiring necrotic tissue removal. In addition, PCD is a mature technology. It is simple and requires less intensive care compared with traditional debridement surgery. The probability of developing complications is lower and it can reduce the fatality rate significantly. It can also be a "guide" for subsequent treatment and save surgery time.

But more study is needed regarding PCD treatment of IPN. For example, when IPN occurs, what types of patients are suitable for PCD treatment and what factors can be used to predict the therapeutic effect of PCD? These kinds of questions are becoming more important recently.

\section{Objectives}

The purpose of this study was to discuss the indicators related to the efficacy of abdominal puncture drainage under CT guidance in patients with acute and severe pancreatitis necrosis tissue infection. To the best of our knowledge, this is the first time that the factors for patients with CT values greater than $20 \mathrm{HU}$ in the puncture area have been analyzed.

\section{Patients and Methods}

\subsection{Individual Patient Data}

This was a retrospective study. The institutional review board in our unit approved this retrospective study with a waiver of informed consent. From January 2013 to November 2016, 162 IPN patients treated with PCD under CT guidance were included in this study. Among them, 92 were male and 70 were female. Etiological classification of patients included 51 cases of cholelithiasis, nine cases of alcoholic pancreatitis, and 11 cases of hyperlipidemia. IPN was confirmed by radiographic examination and the bacterial culture of pancreatic necrotic tissue.
In view of IPN, all patients received no other operation or drainage surgery before the PCD treatment. Depending on the treatment efficacy, 162 patients with severe acute pancreatitis (SAP) were divided into the PCD success treatment group $(n=71)$ and the PCD+ surgery group $(n=91)$.

\subsection{The Treatment of Puncture Drainage Under CT Guidance}

When necrotic pancreatitis and secondary infection was confirmed for the patient, and there was no improvement in the patient's physical condition after conservative treatment or there was even deterioration, PCD treatment was considered. For patients who did not improve after conservative treatment and had no significant deterioration, we usually undergo PCD treatment in about 3 to 4 weeks. Abdominal CT scan or enhanced scan was performed on the patients to find out the location of the focal pancreatic necrosis or fluid and the distance from the surrounding vital organs and large blood vessels. Before treatment, UV disinfection and aseptic treatment were carried out in the CT room. First, progression of pancreatitis was observed by CT plain scan. According to the image, the radiologist and the interventional therapist come to an agreement to find the location and puncture according to the image. Subsequently, body piercing points were selected in the area that was nearest to the drainage area. The best path was chosen through the lateral abdominal wall, the anterior abdominal wall or the posterior abdominal wall. Using puncture and catheter based on a Seldinger puncture technology, Echo 18G puncture needle (Cook Corporation, China) was entered into the area around the pancreas and placed into the drainage tube above 14F. Liquid from the necrotic tissue was immediately drawn for bacterial culture. The whole process of puncture was about 20 minutes. Radiation dose accepted by 162 patients due to CT positioning in puncture process ranged between 20.51 mGy and 25.65 mGy. To prevent drainage pipe blockage, the drainage pipe was flushed every 4 - 8 hours with sterile saline. Each time, as much flush as possible was drawn out of the solid necrotic tissue. After 72 hours of PCD treatment, the drainage efficacy was evaluated based on the assessment of the general situation, the whole abdominal CT scan and the amount of drainage fluid. The standard for clinical improvement was consistent with pancreatitis, necrosectomy versus step-up approach (PANTER) related research (21). If the general situation gradually improved, at least two organs would show improvement (such as the circulatory system, the lungs and the kidneys) or two of three parameters ( $\mathrm{C}$ reactive protein, white blood cells or body temperature) representing improvement of the infection. Flushing through the puncture drainage tube was continued. PCD was done three or more times for some patients on an as-needed basis. If the general physical condition gradually improved, effective flushing through the 
puncture drainage tube was continued and multiple operations of CT-guided PCD were performed when necessary. We pulled out the tube and stopped using antibiotics when the volume of drainage was $<10 \mathrm{~mL}$ for seven consecutive days, or if the CT demonstrated the vomica significantly shrank or disappeared, or if the patients showed no signs of infection such as fever and abdominal pain, or if the blood profile and calcitonin original were normal. At this point, the patient was discharged from the hospital and referred to outpatient follow-ups. These patients were included in the PCD success group.

\subsection{Indicator Monitoring}

Gender, age, body mass index (BMI), acute physiology and chronic health evaluation (APACHEII) scores, c-reactive protein, the CT value of piercing zone, modified CT severity index (MCTSI) scores, the length of time from patient admission to drainage, and the number of tubes were recorded as observation index.

The CT value of the puncture areas was measured in the abdominal CT image by calculating the mean value of CT within the range of $10 \mathrm{~cm}$ diameter using the distal end of the puncture drainage tube as the center.

\subsection{Statistical Analysis}

All statistical analyses were performed with statistical software SPSS statistics for Windows version 17.0 (SPSS Inc., Chicago, Il, USA). The data were in accordance with the normal distribution and the mean value was used. Differences in the different variables of patients were estimated using the $\chi^{2}$ test for categorical data and paired-sample $t$-test for continuous variables. Influencing factors were analyzed using a logistic regression analysis and $\mathrm{P}<0.05$ indicated statistical significance.

\section{Results}

\subsection{Treatment Results}

Overall, 162 patients with AP were included in this study. Among those patients, 92 were male and 70 were female, who were 18 to 75 years old and the mean age was $60.2 \pm 7.8$ years. Seventy-one patients were treated successfully by PCD, while 91 cases showed no remission after PCD treatment and resorted to surgery or laparoscopic surgery. These patients were included in the PCD+ surgery group. The mortality in the PCD+ surgery group was $10.7 \%$. The mortality of the PCD group was $0 \%$. The success rate of PCD treatment was $43.82 \%$.

\subsection{General Conditions of Patients}

There was no significant difference in terms of age, sex, BMI, coexisting diseases, disease severity or organ failure between the two groups (Table 1).
4.3. Comparison of the Therapeutic Effect of Two Groups of Patients

There was a significant statistical difference between the two groups in terms of the time of $C$ reactive protein (CRP) returning to normal, the time of hospitalization, mortality and post treatment complications between the two groups $(\mathrm{P}<0.05)$ (Table 2$)$.

\subsection{Analysis of Factors Influencing the Clinical Efficacy}

Univariate analysis was performed for each observation indicators during the course of treatment. The results showed that the CT value of the puncture area, APACHEII scores and MCTSI scores were related to the therapeutic efficacy $(\mathrm{P}<0.05)$. The multivariate analysis showed that the $\mathrm{CT}$ value of puncture area was the most significant factor with $\mathrm{P}<0.001$ and HR was 11.785 (Table 3 ).

\subsection{Factors Influencing the Clinical Efficacy with Higher CTDen- sity}

We performed further analysis of the factors associated with higher CT density in the puncture area. Some researchers have concluded that when the CT value is less than $20 \mathrm{HU}$, the proportion of the liquid necrosis component is higher than that of the solid necrosis component (11), therefore, in this study, the patients with an average density higher than $20 \mathrm{HU}$ in the CT puncture zone underwent univariate and multivariate analysis. The results showed that the length of time from patient admission to drainage, APACHEII scores, MCTSI scores and C reactive protein levels were significant factors for PCD treatment(Table 4).

\section{Discussion}

There are two important new findings in our study. First, the CT values of the piercing zone is the major risk factor affecting the curative effect of CT guidance PCD. Second, when the average CT density is higher than $20 \mathrm{HU}$, PCD treatment under CT guidance should be performed as soon as possible in order to avoid multiple organ failures or death in the acute stage as well as to avoid a prolonged period of infection.

SAP is a complicated and dangerous condition with a high fatality rate. Past practices have shown that early surgery, debridement surgery and multiple surgeries can increase the incidence of postoperative complications (22). The International Association of Pancreatology (IAP) and the American Pancreatic Association (APA) recommend that the first best treatment for suspected or confirmed pancreatic necrosis should be PCD, and if necessary, followed by endoscopic or debridement operations on the necrotic tissue. The step-up method has been widely 


\begin{tabular}{|c|c|c|c|}
\hline Clinical features & PCD success group & PCD + operation group & Pvalue \\
\hline Gender, male/female & $39 / 32$ & $53 / 38$ & 0.673 \\
\hline Median age, range & $59.23 \pm 7.89$ & $58.34 \pm 8.92$ & 0.345 \\
\hline BMI & $23.23 \pm 2.34$ & $22.34 \pm 3.42$ & 0.765 \\
\hline Cholelithiasis & 51 & 67 & \\
\hline Drinking & 9 & 12 & \\
\hline Hyperlipidemia & 11 & 12 & \\
\hline Comorbidity & & & 0.973 \\
\hline Cardiovascular disease & 37 & 42 & \\
\hline Nephropathy & 12 & 15 & \\
\hline Diabetes mellitus & 25 & 30 & \\
\hline \multicolumn{4}{|l|}{ Disease severity } \\
\hline Leukocyte count (IQR), $10^{9} / \mathrm{L}$ & $7.89 \pm 2.35$ & $8.24 \pm 1.56$ & 0.234 \\
\hline $\mathrm{C}$ reactive protein (IQR), $\mathrm{mg} / \mathrm{L}$ & $187.23 \pm 45.17$ & $174.23 \pm 26.27$ & 0.529 \\
\hline Organ failure & & & 0.972 \\
\hline Failure free & 47 & 60 & \\
\hline Failure & 24 & 31 & \\
\hline
\end{tabular}

Abbreviations: BMI, body mass index; IQR, interquartile range; PCD, percutaneous catheter drainage; SD, standard deviation.

${ }^{\mathrm{a}}$ Values are expressed as No. or mean $\pm \mathrm{SD}$.

\begin{tabular}{|c|c|c|c|}
\hline & PCD success group & PCD + surgery group & Pvalue \\
\hline The time it took for CRP to return to normal & $21.37 \pm 12.36$ & $38.5 \pm 18.53$ & $<0.001$ \\
\hline Days of hospitalization & $35.35 \pm 12.64$ & $65.70 \pm 24.31$ & $<0.001$ \\
\hline Mortality & 0 & 10.7 & $<0.001$ \\
\hline \multicolumn{4}{|l|}{ Post treatment complications } \\
\hline Pancreatic leakage & $3(4.2)$ & $19(21.5)$ & $<0.001$ \\
\hline Intestinal fistula & $4(5.6)$ & $18(19.7)$ & 0.003 \\
\hline Multiple organ failure & $2(2.8)$ & $18(19.7)$ & $<0.001$ \\
\hline Intraperitoneal hemorrhage & $3(4.2)$ & $17(19.5)$ & $<0.001$ \\
\hline
\end{tabular}

Abbreviations: CRP, C reactive protein; d, days; $\mathrm{PCD}$, percutaneous catheter drainage; $\mathrm{SD}$, standard deviation

${ }^{\mathrm{a}}$ Values are expressed as No.(\%) or mean \pm SD.

recognized $(21,23)$. PCD is the initial step of the step-up method and is the core of the entire treatment. Some reports have demonstrated significant difference in terms of the success rate in PCD treatment of IPN (23). A retrospective study conducted by Sileikis et al. concluded that stepup approach is not always effective for patients with multiple organ failure, which is only about $50 \%$ success rate, and they suggest that surgery should not be delayed for more than one month (24).

In our research, we found that the patients who were successfully treated by PCD were shorter in hospital time and CRP recovery time than those who had PCD combined surgery. We also found that the mortality rate was low, and the incidence of postoperative multiple organ failure was low. So, are there any factors that can predict the effect of PCD before treatment? Are there any measures that can improve the success rate of PCD therapy? It is mainly due to the different body status, the duration of IPN and the degree of necrosis liquefaction in different patients. These factors may significantly influence the successful rate of 


\begin{tabular}{|c|c|c|c|c|c|}
\hline \multirow[t]{2}{*}{ Clinical features } & \multirow[t]{2}{*}{ PCD success group } & \multicolumn{2}{|c|}{ Univariate analysis } & \multicolumn{2}{|c|}{ Multivariate analysis } \\
\hline & & Chi-square value & Pvalue & HR & Pvalue \\
\hline Gender & & 0.178 & 0.673 & & \\
\hline Male & 39 & & & & \\
\hline Female & 32 & & & & \\
\hline Age, $y$ & & 0.239 & 0.625 & & \\
\hline$\leq 60$ & 30 & & & & \\
\hline$>60$ & 41 & & & & \\
\hline Cholelithiasis & & 0.065 & 0.799 & & \\
\hline Yes & 51 & & & & \\
\hline No & 20 & & & & \\
\hline Number of puncture catheterizations, root & & 2.837 & 0.092 & & \\
\hline$\leq 3$ & 43 & & & & \\
\hline$>3$ & 28 & & & & \\
\hline Puncture zone CT value, $\mathrm{HU}$ & & 6.247 & 0.012 & 11.785 & $<0.001$ \\
\hline$\leq 20$ & 46 & & & & \\
\hline$>20$ & 25 & & & & \\
\hline $\begin{array}{l}\text { Length of time from patient admission to drainage, } \\
\text { d }\end{array}$ & & 1.186 & 0.553 & & \\
\hline$\leq 9$ & 18 & & & & \\
\hline $9-14$ & 31 & & & & \\
\hline$>14$ & 22 & & & & \\
\hline APACHEII score & & 8.235 & 0.016 & 0.236 & $<0.001$ \\
\hline$\leq 8$ & 19 & & & & \\
\hline $8-10$ & 18 & & & & \\
\hline$>10$ & 34 & & & & \\
\hline MCTSI score & & 10.049 & 0.007 & 2.414 & $<0.001$ \\
\hline$\leq 3$ & 20 & & & & \\
\hline $4-6$ & 31 & & & & \\
\hline $7-10$ & 20 & & & & \\
\hline$C$ reactive protein & & 2.194 & 0.334 & & \\
\hline$\leq 150$ & 13 & & & & \\
\hline $150-300$ & 22 & & & & \\
\hline$>300$ & 36 & & & & \\
\hline
\end{tabular}

Abbreviations: APACHE II, acute physiology and chronic health evaluation; d, days; HR, hazard ratio; HU, Hounsfield unit; MCTIS, modified CT severity index; PCD, percutaneous catheter drainage; $y$, years

PCD treatment. The current international guidelines do not provide specific guidance regarding PCD treatment in the step-up regimen $(8,25)$, there is no uniform consensus in terms of which factors will affect the efficacy of PCD treatment.

PCD treatment can remove the necrotic materials while draining the effusion at the same time $(26,27)$, and solid materials can be drawn from tissues of IPN. However, before the PCD operation, we could predict the proportions of solid and liquid materials by observing the average CT density in the pre-piercing zone on the CT scanning image. This time we mainly study and analyze the treatment of IPN under the guidance of CT. Spiral CT collects the volume data, can carry on the three-dimensional and 


\begin{tabular}{|c|c|c|c|c|c|}
\hline \multirow[t]{2}{*}{ Clinical features } & \multirow[t]{2}{*}{ PCD success } & \multicolumn{2}{|c|}{ Univariate analysis } & \multicolumn{2}{|c|}{ multivariate analysis } \\
\hline & & Chisquarevalue & Pvalue & HR & Pvalue \\
\hline Gender & & 2.280 & 0.131 & & \\
\hline Male & 23 & & & & \\
\hline Female & 2 & & & & \\
\hline Age, $y$ & & 3.046 & 0.065 & & \\
\hline$\leq 60$ & 6 & & & & \\
\hline$>60$ & 19 & & & & \\
\hline Cholelithiasis & & 2.749 & 0.097 & & \\
\hline Yes & 18 & & & & \\
\hline No & 7 & & & & \\
\hline The number of puncture tubes, root & & 0.712 & 0.399 & & \\
\hline$\leq 3$ & 14 & & & & \\
\hline$>3$ & 11 & & & & \\
\hline length of time from patient admission to drainage, $d$ & & 7.013 & 0.030 & 11.162 & 0.007 \\
\hline$\leq 9$ & 7 & & & & \\
\hline $9-14$ & 15 & & & & \\
\hline$>14$ & 3 & & & & \\
\hline APACHEII score & & 17.459 & $<0.001$ & 0.030 & 0.002 \\
\hline$\leq 8$ & 0 & & & & \\
\hline $8-10$ & 2 & & & & \\
\hline$>10$ & 23 & & & & \\
\hline MCTSI score & & 40.969 & $<0.001$ & 17.262 & 0.001 \\
\hline$\leq 3$ & 19 & & & & \\
\hline $4-6$ & 0 & & & & \\
\hline 7-10 & 6 & & & & \\
\hline CRP & & 7.534 & 0.023 & 0.047 & 0.004 \\
\hline$\leq 150$ & 0 & & & & \\
\hline $150-300$ & 17 & & & & \\
\hline$>300$ & 8 & & & & \\
\hline
\end{tabular}

Abbreviations: APACHE II, acute physiology and chronic health evaluation; CRP, C reactive protein; d, days; HR, hazard ratio; HU, Hounsfield unit; MCTIS, modified CT severity index; $P C D$, percutaneous catheter drainage; $y$, years

multi-directional observation, the location is accurate, and the guide puncture is more precise. According to the diagnostic criteria of the Atlanta conference in 1992, (1) infective pancreatic necrosis must have solid sphacelus components, but not completely liquefied solid sphacelus had higher CT values, similar to soft tissue density. Therefore, a higher value of $\mathrm{CT}$ density average indicates a higher content of solid necrosis or blood clot. These results showed that when the average $\mathrm{CT}$ value was higher, the chance of PCD failure was also higher, accompanied by a lower cure rate. In our study, only $35.2 \%$ of patients in the PCD success group had a CT value greater than $20 \mathrm{HU}$ in the puncture area. (Figures 1 and 2).These results were consistent with the results from Tong et al.'s research (11).

In 2004, Mortele et al. suggested that the SAP computed tomography severity index, namely MCTSI, reflected organ failure situation and pancreatic complications, as shown by the amount of effusion. The range of pancreatic necrosis was reduced to less than $30 \%$ and more than $30 \%$. In MCTSI, the non-pancreas complications were also considered, including peri-pancreatic blood vessel invasion and gastrointestinal tract invasion. Recent studies con- 

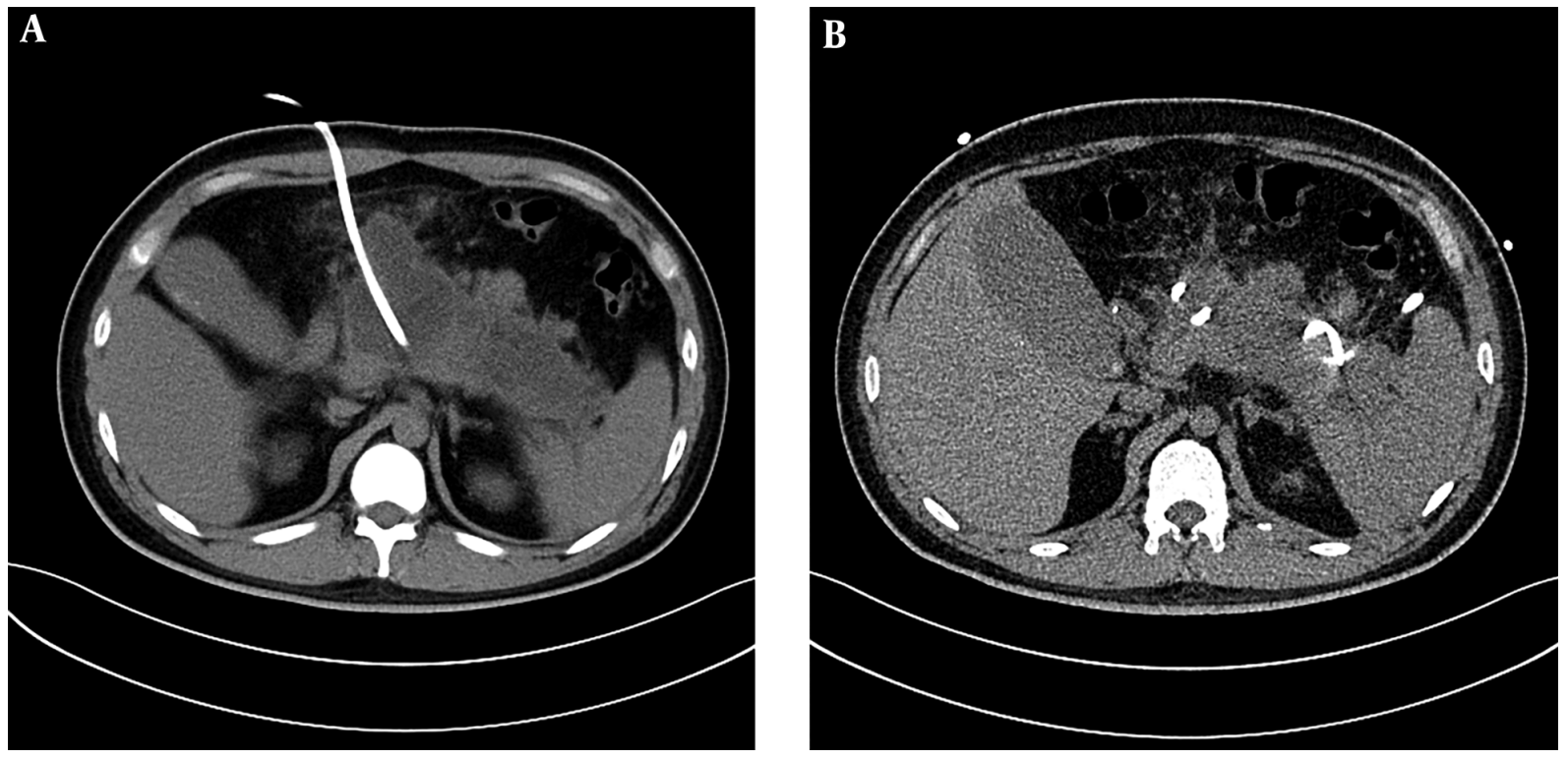

Figure 1. A 36-year-old man with acute necrotizing pancreatitis accompanied by acute abdominal pain, vomiting and fever. A, The liquefied area of pancreatic necrosis underwent CT guided puncture and the average CT value of the piercing area was about 10 Hounsfield unit (HU) after successful puncturing and cathetering, and effective flushing through the puncture drainage tube was continued. B, In a month, re-examined abdominal CT and the low density pancreas area reduced obviously. The patient recovered gradually and no open operation was required.
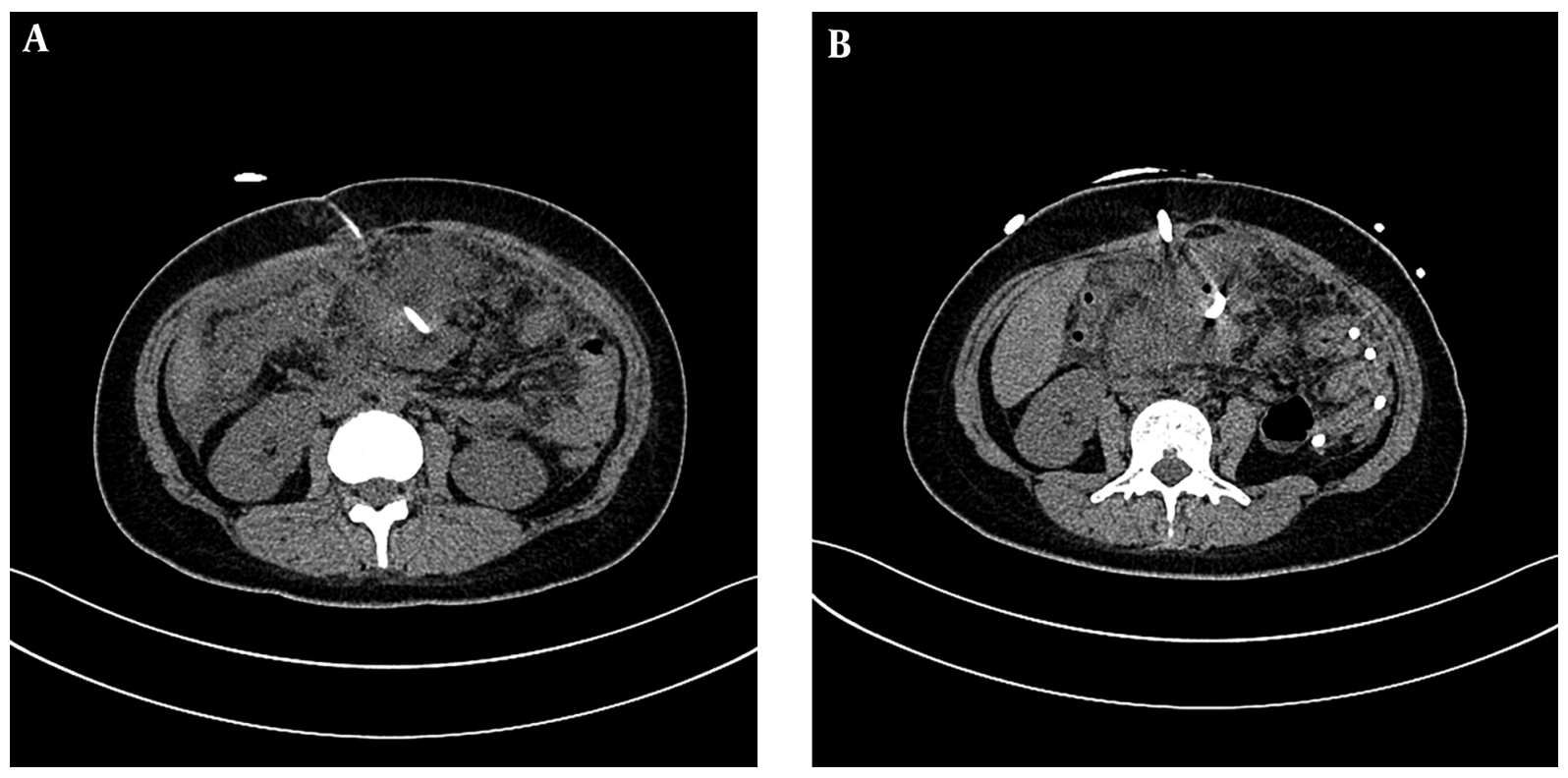

Figure 2. A 66-year-old man with acute necrotizing pancreatitis. A, Under CT guided puncture, the density around the pancreas leakage was higher and the average CT score was more than 20 Hounsfield unit (HU). B, A week later, CT examination was performed and the leakage around the pancreas was more apparent. The patient's condition was poor and an open debridement surgery was eventually performed.

firmed that the correlation between MCTSI and the prognosis was good (28). The present study found that MCTSI index had a good correlation with the curative efficacy of PCD, and the higher the MCTSI index, the higher chance of
PCD failure. There are 12 monitoring indicators in APACHE II and if the severity and prognosis of AP can be predicted early, it may significantly improve the treatment efficacy. This scoring system could assess the severity of the disease 
repeatedly at any time during and after hospitalization. Our study found that APACHE II was also one of the independent risk factors for PCD efficacy.

In addition, the length of time from patient admission to drainage is important for the success of the treatment. A team of researchers suggested that if there was no technical difficulty, PCD should be performed relatively early to reduce the incidence of complications and to shorten the length of hospital stay (29). The authors evaluated the positive and conventional PCD treatment in patients with infectious necrotizing pancreatitis, and found that an active PCD therapy can reduce the need for surgical necrosectomy (30). PCD treatment was less effective in patients with a higher CT value in the puncture area, but what factors could reflect the efficacy of the treatment on a deeper level? In our study, we analyzed the patients who had a CT value greater than $20 \mathrm{HU}$ in the puncture area and were successful in the PCD treatment, about 12\% of patients received PCD treatment in more than 14 days and most patients (60\%) were treated with PCD between 9 days and 14 days. Our results suggested that the first PCD treatment should not be too late. This indicates that when CT images showed a large amount of exudation in the retroperitoneum and peritoneal cavity, the average CT density is higher. After conservative treatment, if the patient's condition did not improve, PCD treatment under CT guidance should be performed as soon as possible in order to avoid multiple organ failures or death in the acute stage as well as to avoid a prolonged period of infection. When the drainage does not smoothen, we can use a coarser drainage tube or a three-cavity lavage drainage tube promptly. In addition, multiple ports could be maintained for possible follow-up endoscopic removal of necrotic tissues.

CRP is a kind of acute reactive proteins synthesized by the liver. It is mainly produced during the inflammation and acts against the harmful effect of soluble protease released from the trauma and infection site $(31,32)$. The increase of CRP is one of the acute phase reactions in the body when infection and injury occur $(33,34)$. It is present in serum and reaches a peak level in 24 - 48 hours after the onset of disease. Schutte and Malfertheiner (32) reported that CRP levels are proportional to the severity of AP. Furthermore, it has important clinical values regarding early disease diagnosis, complications and prognosis (35). Our results showed that CRP is an independent risk factor in the PCD therapy and patients with a higher CRP level have a poor PCD efficacy.

This study is a single center retrospective analysis, so there may be some empiricism and blindness. Multicenter, prospective, randomized and controlled clinical trials with large sample sizes are required to determine the efficacy of PCD and relevant influencing factors in the future.
In conclusion, our study found that the average CT value from the infection of pancreatic necrotic tissue is a major risk factor for PCD treatment under CT guidance, and the higher the CT value, the higher possibility of treatment failure. The length of time from patient admission to drainage, APACHEII scores, MCTSI scores and $\mathrm{C}$ reactive protein levels should be evaluated comprehensively. We think that in patients with an average density higher than $20 \mathrm{HU}$ in the CT puncture zone, PCD treatment should be performed as soon as possible.

\section{Footnotes}

Authors' Contributions: Xuening Zhang chose the direction of the subject, and Xin Xu gathered clinical data and was responsible for general writing clinical imaging and diagnosis, and revision of the manuscript.

Conflicts of Interests: The authors have no conflicts of interest to declare.

Ethical Considerations: This was a retrospective study. The institutional review board in our unit approved this retrospective study with a waiver of informed consent.

Financial Disclosure: Our research had no relevant financial problems.

Funding/Support: No financial support.

\section{References}

1. Banks PA, Bollen TL, Dervenis C, Gooszen HG, Johnson CD, Sarr MG et al. Classification of acute pancreatitis-2012: Revision of the Atlanta classification and definitions by international consensus. Gut. 2013;62(1):102-11. doi: 10.1136/gutjnl-2012-302779. [PubMed: 23100216].

2. Chen H, Li F, Sun JB, Jia JG. Abdominal compartment syndrome in patients with severe acute pancreatitis in early stage. World $J$ Gastroenterol. 2008;14(22):3541-8. doi: 10.3748/wjg.14.3541. [PubMed: 18567084]. [PubMed Central: PMC2716618].

3. Whitcomb DC. Clinical practice. Acute pancreatitis. $N$ Engl J Med. 2006;354(20):2142-50. doi: 10.1056/NEJMcp054958. [PubMed: 16707751].

4. Rau B, Bothe A, Beger HG. Surgical treatment of necrotizing pancreatitis by necrosectomy and closed lavage: Changing patient characteristics and outcome in a 19-year, single-center series. Surgery. 2005;138(1):28-39. doi: 10.1016/j.surg.2005.03.010. [PubMed: 16003313].

5. Rodriguez JR, Razo AO, Targarona J, Thayer SP, Rattner DW, Warshaw AL, et al. Debridement and closed packing for sterile or infected necrotizing pancreatitis: Insights into indications and outcomes in 167 patients. Ann Surg. 2008;247(2):294-9. doi: 10.1097/SLA.0b013e31815b6976. [PubMed: 18216536]. [PubMed Central: PMC3806106].

6. Banks PA, Freeman ML, Practice Parameters Committee of the American College of $G$. Practice guidelines in acute pancreatitis. Am J Gastroenterol. 2006;101(10):2379-400. doi: 10.1111/j.15720241.2006.00856.x. [PubMed: 17032204].

7. Petrov MS, Shanbhag S, Chakraborty M, Phillips AR, Windsor JA. Organ failure and infection of pancreatic necrosis as determinants of mortality in patients with acute pancreatitis. Gastroenterology. 2010;139(3):813-20. doi: 10.1053/j.gastro.2010.06.010. [PubMed: 20540942] 
8. Tenner S, Baillie J, DeWitt J, Vege SS, American College of G. American College of Gastroenterology guideline: Management of acute pancreatitis. Am J Gastroenterol. 2013;108(9):1400-15. 1416. doi: 10.1038/ajg.2013.218. [PubMed: 23896955].

9. Yokoe M, Takada T, Mayumi T, Yoshida M, Isaji S, Wada K, et al. Japanese guidelines for the management of acute pancreatitis: Japanese Guidelines 2015. J Hepatobiliary Pancreat Sci. 2015;22(6):405-32. doi: 10.1002/jhbp.259. [PubMed: 25973947].

10. Sleeman D, Levi DM, Cheung MC, Rahnemai-Azar A, Parisek S, Casillas $\mathrm{V}$, et al. Percutaneous lavage as primary treatment for infected pancreatic necrosis. J Am Coll Surg. 2011;212(4):748-52. discussion 752-4. doi: 10.1016/j.jamcollsurg.2010.12.019. [PubMed: 21463827].

11. Tong Z, Li W, Yu W, Geng Y, Ke L, Nie Y, et al. Percutaneous catheter drainage for infective pancreatic necrosis: Is it always the first choice for all patients? Pancreas. 2012;41(2):302-5. doi: 10.1097/MPA.0b013e318229816f. [PubMed: 21926935].

12. Wronski M, Cebulski W, Karkocha D, Slodkowski M, Wysocki L, Jankowski M, et al. Ultrasound-guided percutaneous drainage of infected pancreatic necrosis. Surg Endosc. 2013;27(8):2841-8. doi: 10.1007/s00464-013-2831-9. [PubMed: 23404151]. [PubMed Central: PMC3710405].

13. van Baal MC, van Santvoort HC, Bollen TL, Bakker OJ, Besselink MG, Gooszen HG, et al. Systematic review of percutaneous catheter drainage as primary treatment for necrotizing pancreatitis. BrJ Surg. 2011;98(1):18-27. doi: 10.1002/bjs.7304. [PubMed: 21136562].

14. Echenique AM, Sleeman D, Yrizarry J, Scagnelli T, Guerra JJ Jr, Casillas VJ, et al. Percutaneous catheter-directed debridement of in fected pancreatic necrosis: Results in 20 patients. J Vasc Interv $R a$ diol. 1998;9(4):565-71. doi: 10.1016/S1051-0443(98)70323-9. [PubMed: 9684824].

15. Szentkereszty Z, Kerekes L, Hallay J, Czako D, Sapy P. CT-guided percutaneous peripancreatic drainage: A possible therapy in acute necrotizing pancreatitis. Hepatogastroenterology. 2002;49(48):16968. [PubMed: 12397769].

16. Lee JK, Kwak KK, Park JK, Yoon WJ, Lee SH, Ryu JK, et al. The efficacy of nonsurgical treatment of infected pancreatic necrosis. Pancreas. 2007;34(4):399-404. doi: 10.1097/MPA.0b013e318043c0b1. [PubMed: 17446837].

17. Rocha FG, Benoit E, Zinner MJ, Whang EE, Banks PA, Ashley SW, et al. Impact of radiologic intervention on mortality in necrotizing pancreatitis: The role of organ failure. Arch Surg. 2009;144(3):261-5. doi: 10.1001/archsurg.2008.587. [PubMed: 19289666].

18. Baudin G, Chassang M, Gelsi E, Novellas S, Bernardin G, Hebuterne X, et al. CT-guided percutaneous catheter drainage of acute infectious necrotizing pancreatitis: Assessment of effectiveness and safety. AJR Am J Roentgenol. 2012;199(1):192-9. doi: 10.2214/AJR.11.6984. [PubMed: 22733912].

19. Solanki R, Thumma V, Sastry RA, Bheerappa N. The role of image guided percutaneous drainage in multidisciplinary management of necrotizing pancreatitis. Trop Gastroenterol. 2013;34(1):25-30. doi 10.7869/tg.2012.87. [PubMed: 23923371].

20. Babu RY, Gupta R, Kang M, Bhasin DK, Rana SS, Singh R. Predictors of surgery in patients with severe acute pancreatitis managed by the step-up approach. Ann Surg. 2013;257(4):737-50. doi 10.1097/SLA.0b013e318269d25d. [PubMed: 22968079].

21. van Santvoort HC, Besselink MG, Bakker OJ, Hofker HS, Boermeester $\mathrm{MA}$, Dejong $\mathrm{CH}$, et al. A step-up approach or open necrosectomy for necrotizing pancreatitis. N Engl J Med. 2010;362(16):1491-502. doi: 10.1056/NEJMoa0908821. [PubMed: 20410514].
22. Bradley EL 3rd, Dexter ND. Management of severe acute pancreatitis: A surgical odyssey. Ann Surg. 2010;251(1):6-17. doi: 10.1097/SLA.0b013e3181c72b79. [PubMed: 20009748].

23. da Costa DW, Boerma D, van Santvoort HC, Horvath KD, Werner J, Carter CR, et al. Staged multidisciplinary step-up management for necrotizing pancreatitis. Br J Surg. 2014;101(1):e65-79. doi: 10.1002/bjs.9346. [PubMed: 24272964]

24. Sileikis A, Peciulyte E, Misenkiene A, Klimasauskas A, Beisa V, Strupas $K$. Is minimally invasive surgical treatment justified for severe acute necrotizing pancreatitis patients with dysfunction of two or more organ systems? Wideochir Inne Tech Maloinwazyjne. 2017;12(3):225-30. doi: 10.5114/wiitm.2017.68792. [PubMed: 29062441]. [PubMed Central: PMC5649498].

25. Working Group IAP/APA Acute Pancreatitis Guidelines. IAP/APA evidence-based guidelines for the management of acute pancreatitis. Pancreatology. 2013;13(4 Suppl 2):e1-15. doi: 10.1016/j.pan.2013.07.063. [PubMed: 24054878].

26. Lau ST, Simchuk EJ, Kozarek RA, Traverso LW. A pancreatic ductal leak should be sought to direct treatment in patients with acute pancreatitis. Am J Surg. 2001;181(5):411-5. doi: 10.1016/S0002-9610(01)00606-7. [PubMed: 11448431]

27. Sugimoto M, Sonntag DP, Flint GS, Boyce CJ, Kirkham JC, Harris TJ, et al. A percutaneous drainage protocol for severe and moderately severe acute pancreatitis. Surg Endosc. 2015;29(11):3282-91. doi: 10.1007/s00464-015-4077-1. [PubMed: 25631111].

28. Jain S, Gupta S, Chawla AS, Agarwal Y, Thukral BB. Comparative study of balthazar computed tomography severity index and modified computed tomography severity index in predicting the outcome of acute pancreatitis. Apollo Med. 2014;11(2):74-83. doi: 10.1016/j.apme.2014.03.002.

29. van Grinsven J, van Santvoort HC, Boermeester MA, Dejong CH, van Eijck $\mathrm{CH}$, Fockens $\mathrm{P}$, et al. Timing of catheter drainage in infected necrotizing pancreatitis. Nat Rev Gastroenterol Hepatol. 2016;13(5):306-12. doi: 10.1038/nrgastro.2016.23. [PubMed: 26956064].

30. van Grinsven J, Timmerman P, van Lienden KP, Haveman JW, Boerma $\mathrm{D}$, van Eijck $\mathrm{CH}$, et al. Proactive versus standard percutaneous catheter drainage for infected necrotizing pancreatitis. Pancreas. 2017;46(4):518-23. doi: 10.1097/MPA.0000000000000785. [PubMed: 28196022].

31. Riche FC, Cholley BP, Laisne MJ, Vicaut E, Panis YH, Lajeunie EJ, et al. Inflammatory cytokines, $\mathrm{C}$ reactive protein, and procalcitonin as early predictors of necrosis infection in acute necrotizing pancreatitis. Surgery. 2003;133(3):257-62. doi: 10.1067/msy.2003.70. [PubMed: 12660636].

32. Schutte $\mathrm{K}$, Malfertheiner $\mathrm{P}$. Markers for predicting severity and progression of acute pancreatitis. Best Pract Res Clin Gastroenterol. 2008;22(1):75-90. doi: 10.1016/j.bpg.2007.10.013. [PubMed: 18206814].

33. Pearce CB, Gunn SR, Ahmed A, Johnson CD. Machine learning can improve prediction of severity in acute pancreatitis using admission values of APACHE II score and C-reactive protein. Pancreatology. 2006;6(1-2):123-31. doi:10.1159/000090032. [PubMed: 16327290].

34. Al-Bahrani AZ, Ammori BJ. Clinical laboratory assessment of acute pancreatitis. Clin Chim Acta. 2005;362(1-2):26-48. doi: 10.1016/j.cccn.2005.06.008. [PubMed: 16024009].

35. Kompoti M, Drimis S, Papadaki A, Kotsomytis K, Poulopoulou C, Gianneli D, et al. Serum C-reactive protein at admission predicts in-hospital mortality in medical patients. Eur J Intern Med. 2008;19(4):261-5. doi: 10.1016/j.ejim.2007.06.016. [PubMed: 18471674]. 